\title{
Encuestas presenciales con cuestionario de papel y ordenador. Una comparativa en preguntas de actitudes*
}

\author{
Vidal Díaz de Rada \\ Universidad Pública de Navarra \\ vidal@unavarra.es
}

\section{Resumen}

En este trabajo se compara la calidad de las respuestas obtenidas por un mismo cuestionario que es administrado en papel y con ordenador en dos muestras equivalentes. La medición de la calidad se realizará considerando el promedio de preguntas no respondidas en cada modalidad, así como el número de "no sabe» y "no responde» logrado por las preguntas de respuesta única y las multirrespuestas. El análisis de todo el cuestionario desvela que el número de "no sabe» es superior al de «no responde», pese a que el segundo es mayor en el cuestionario realizado en papel. En las preguntas de elección condicionada, de contestación única, el número de no respuestas es superior a los «no sabe» y aumentan sustancialmente en el cuestionario de papel. De las seis preguntas multirrespuesta solo tres presenta diferencias significativas, aunque los resultados no son concluyentes.

Palabras clave: encuesta personal; encuesta con ordenador; modos de recogida de información; efectos de respuesta

\section{Abstract. Face-to-face surveys using paper or a computer: Difference in attitudes}

This article examines the implications for data quality of using computer-assisted personal interviewing (CAPI) compared to paper-and-pencil interviewing (PAPI) in an electoral survey. Quality is measured by considering the average of unanswered questions in each modality and the number of non-responses in single-answer multiple choice questions. The analysis of the questionnaire as a whole reveals that the number of "don't know" responses is higher than the "no answer" option, although the second is more frequent in PAPI. In forced choice questions requiring a single answer, the number of non-responses is greater than the "don't know" responses; a figure that increases in PAPI. Of the six multiple choice questions, only three show significant differences, although the results are inconclusive.

Keywords: face-to-face survey; computer-assisted interview; data collection modes; response effects

* Este texto es parte de una investigación financiada por el Ministerio de Economía y Competitividad, referencia MTM2015-63609-R. El autor desea agradecer la mejora del trabajo original propuesto por el Consejo Editorial de Papers y por dos evaluadores anónimos. Agradezco también el asesoramiento estadístico realizado por Antonio Pardo, de la Universidad Autónoma de Madrid. 


\section{Sumario}

1. Encuesta presencial asistida por ordenador

2. La situación en España

3. Diseño de la investigación: estudio postelectoral de las elecciones autonómicas y municipales (2011) en la Comunidad de Madrid (estudios CIS 2893 y 2894)

4. Resultados
5. Conclusiones y discusión

Referencias bibliográficas

Anexo 1. Temática de las preguntas del cuestionario (estudios 2893 y 2894)

Anexo 2. Distribución muestral considerando sexo, edad, tipo de familia, nivel de estudios, relación con la actividad, situación profesional y clase social (autoadscripción)

La encuesta presencial es el modo de recogida de datos mediante el cual un entrevistador administra un cuestionario estructurado (o parcialmente estructurado) frente a un entrevistado dentro de un período de tiempo limitado, generalmente en su hogar (De Leeuw, 2004). La aplicación de avances informáticos a la disciplina desarrolla la entrevista computerizada (Saris, 1991), que busca «integrar y automatizar al máximo el proceso de encuesta». De este modo, la recogida de datos está unida a la introducción de los mismos, con lo que se realizan simultáneamente tareas que antes eran efectuadas por separado. La primera aplicación de la encuesta personal asistida por ordenador (en adelante CAPI) se llevó a cabo en la encuesta de población activa (Netherlands Labour Force Survey) de los Países Bajos (Van Bastelaer et al., 1988). Se trata de una modalidad que se ha desarrollado fundamentalmente en Europa (Bergman et al., 1994), probablemente por el menor uso de la encuesta telefónica (Martin y Manners, 1995).

El objetivo de este trabajo es comparar la calidad de la información recogida mediante dos modalidades ${ }^{1}$ (cuestionario de papel y cuestionario en ordenador), utilizando una investigación realizada por el Centro de Investigaciones Sociológicas (en adelante CIS) a la población con derecho a voto y residente en la Comunidad Autónoma de Madrid en mayo y junio de 2011. Se trata, concretamente, de los estudios 2893 y 2894 (CIS, 2011a y 2011b).

La medición de la calidad se llevará a cabo considerando el promedio de preguntas no respondidas en los cuestionarios realizados con cada modalidad, tema de una gran importancia en la investigación con encuesta, no solo por la pérdida (o no recogida) de una información en ocasiones muy valiosa y fundamental (Tabachnick y Fidell, 1989), sino también por las implicaciones que genera en el análisis de datos, especialmente cuando se trabaja con técnicas multivariantes. Bourque y Clark (1992) estiman que una regresión múltiple

1. El término modalidades se ha referido tradicionalmente a las encuestas presenciales, telefónicas y autoadministradas, aunque en las publicaciones más recientes se emplea también para las variantes de estas, como las encuestas telefónicas automáticas, las encuestas autoadministradas, etc. En el presente trabajo, dicho término será utilizado para comparar la encuesta presencial tradicional (papel) con la que utiliza un ordenador (CAPI). 
con 25 variables, en la que cada una tuviera un $2 \%$ de casos sin respuesta, podría generar una reducción del $40 \%$ en el tamaño muestral total. En esta misma línea, Roth (1994) señala que la eliminación aleatoria del 10\% de los casos de cada variable en una matriz de cinco variables implicaría perder el 59\% de los casos.

El presente trabajo se ha estructurado en cinco partes, comenzando por una exposición de las ventajas del sistema CAPI frente al papel tradicional, sintetizada tras la revisión de la mayor parte de la literatura sobre el tema. Seguidamente hay un breve apartado sobre la situación en España, que plantea algunos interrogantes sobre el gran retraso de la investigación española en la adopción de esta tecnología. A continuación se describe la fuente de datos utilizada detallando la metodología y los objetivos de la investigación. En el cuarto apartado se presentan los resultados considerando el número total de no respuestas, la ausencia de respuesta en preguntas de respuesta única, el número de respuestas conseguidas en las preguntas de respuesta múltiple y las no respuestas en las preguntas finales del cuestionario. Esta exposición precede a las conclusiones.

\section{Encuesta presencial asistida por ordenador}

La característica esencial de esta modalidad es la sustitución del tradicional —y rígido- cuestionario (de papel) por un ordenador portátil, en cuya pantalla van apareciendo las diferentes preguntas del cuestionario (De Leeuw y Nicholls II, 1996; Couper y Hansen, 2002). Así, en vez de registrar las respuestas en el papel, la recogida de datos está unida a la introducción de los mismos, con lo que ambas tareas se realizan simultáneamente.

Es un modo de recogida de datos muy similar a la encuesta telefónica asistida por ordenador que permite aleatorizar las categorías de respuesta, disponer de ayudas a la codificación de preguntas abiertas, filtrar preguntas, etc. (De Leeuw y Nicholls II, 1996; Martin y Manners, 1995). Por ello se considera que la mayor ventaja del CAPI es que el entrevistador se concentra en el proceso de la entrevista, logrando así más interacción con el entrevistado (De Leeuw, 2004). Una de las mayores ventajas es que el ordenador realiza automáticamente los pertinentes «saltos» de preguntas originados por las "preguntas filtro", con lo que aumenta la calidad de la información recogida. Investigaciones realizadas en otros contextos dan cuenta de que el empleo del CAPI — frente al papel — supone una importante reducción de «saltos erróneos»: el 0,97\% del cuestionario de papel se reduce al 0,02\% en el cuestionario CAPI según Olsen (1992), mientras que otros sitúan estas cifras en el 4,6\% y el 1,8\% (Couper y Hansen, 2002). La complejidad del cuestionario explica las diferencias entre unos estudios y otros, aunque existe un acuerdo unánime en que esta es una de las principales ventajas del CAPI frente al cuestionario de papel.

Otras mejoras no menos importantes están relacionadas con la disposición de determinados controles de consistencia ("preguntas de control») incluidos a lo largo del cuestionario que permiten localizar respuestas inconsistentes (Van Bastelaer et 
al., 1988), al indicar al entrevistador que vuelva a realizar determinadas preguntas o, en su caso, que verifique la exactitud de ciertas respuestas (Couper y Hansen, 2002; Martin y Manners, 1995).

Este sistema también lleva a cabo un mayor control del trabajo de campo: el registro automático de la hora de comienzo y finalización de la entrevista permite conocer el ritmo de trabajo del entrevistador, si este cumple — por ejemplo— la recomendación del coordinador de campo de realizar entrevistas durante todo el día, al tiempo que proporciona registros precisos sobre el tiempo medio para responder al cuestionario. Esto último es esencial para realizar controles exhaustivos de las entrevistas «muy cortas» $\mathrm{o}$ "muy largas». El propio sistema, en función de las normas de la empresa, puede evitar que los entrevistadores editen la encuesta, siendo "cerradas» una vez terminada la entrevista (De Leeuw y Nicholls II, 1996; Hernández Moreno et al., 2011).

Evidentemente, no todo son ventajas. Existen algunos inconvenientes relacionados con el instrumento de recogida de datos (ordenador portátil o PDA), debido a que la pantalla tiene un menor tamaño que el papel, lo que puede producir errores en la grabación de las respuestas, principalmente cuando se trata de preguntas abiertas, aunque diversas investigaciones han demostrado que los errores por este motivo son mínimos. Así, tras revisar 16.000 cuestionarios, Dielman y Couper (1995) localizan una tasa de error del 0,095\%, cifra que Kennedy et al. (1990) aumentan al 0,63\%, y Lepkowski et al. (1995) al $1 \%$ en varias oleadas del Panel of Income Dyamics. Otros problemas están relacionados con el hecho de que los entrevistados pueden reaccionar negativamente ante la presencia del ordenador, pese a que diversas investigaciones (entre otros, CIS, 2012; De Leeuw y Collins, 1997; Lamas, 2002, 2003) han demostrado que esta reacción negativa es mínima y que ejerce poca influencia sobre la tasa de respuesta. Así, y aunque los entrevistadores que han participado en el estudio piloto del CIS consideran que un $60 \%$ de los entrevistados prefieren responder en papel (CIS, 2012), Baker et al. (1995) localizan sensaciones negativas únicamente en el $5 \%$ de los entrevistados, cifras que De Leeuw et al. (1995) reducen hasta el 1\%-4\%. Otros autores, como Martin y Manners (1995), dan cuenta de la indiferencia de los entrevistados en cuanto al uso de un ordenador durante la entrevista.

¿Cómo afecta este tiempo al coste total de la investigación realizada? Al ahorro de papel y fotocopias (o impresión del cuestionario) se contrapone el tiempo y el coste de «convertir» el cuestionario de "papel» en un cuestionario «visual». Este proceso no solo requiere una gran cantidad de tiempo, sino que además deberá ser realizado por personal especializado que generará un notable incremento en el coste total de la investigación. Así, por ejemplo, podemos indicar que el cambio de papel a CAPI del Estudio General de Medios supuso un aumento económico del 12\% (Lamas, 2003). El ahorro en la introducción de datos es notable, aunque este es uno de los aspectos más económicos dentro del proceso de investigación con encuesta. No obstante, esta modalidad consigue una adecuada relación entre calidad y coste con grandes tamaños muestrales, puesto que con grandes muestras la menor duración de cada entre- 
vista genera un importante ahorro de tiempo, y el coste de la preparación del cuestionario virtual queda más repartido entre todos los cuestionarios. Esto explica que este sistema de recogida de datos sea utilizado frecuentemente en las encuestas de panel, en las que un mismo cuestionario es aplicado durante un gran período de tiempo (Lamas, 2003; Schräpler et al., 2010; Lynn, 2016). Pese a este mayor coste, más reducido actualmente por el descenso en el precio de los equipos informáticos, el argumento fundamental para utilizar el CAPI es la mejora en la calidad de los datos recogidos (Baker et al., 1995; Schräpler et al., 2010): menos «contaminación» de unas preguntas por otras, menor índice de preguntas no respondidas, menos «deseabilidad social» en las respuestas, verificación de la coherencia del entrevistado a través de numerosos filtros, mayor estandarización de la entrevista, etc.

\section{La situación en España}

Vistas las mejoras y los inconvenientes que supone la utilización del sistema CAPI para optimizar la calidad de la respuesta, es el momento de analizar la implantación de este dispositivo en la investigación que tiene lugar en España. En unos encuentros del CIS realizados a principios del presente siglo, Jorge Clemente señalaba que una de las innovaciones más importantes que experimentará la encuesta presencial será la incorporación de los ordenadores portátiles, dando cuenta de que — en esos momentos - más de la mitad de los encuestadores de su empresa (Eco-Consulting) trabajaba con este equipamiento y destacando que esta será una de las áreas con más desarrollo futuro (CIS, 2002). Ahora bien, la estimación realizada por AEDEMO en el estudio sobre la industria de los estudios de mercado, mostrada en la tabla 1 , desvela que esta predicción ha tardado en hacerse realidad.

La primera referencia al número de encuestas realizadas con CAPI tiene lugar en la edición del año 2003, donde se señala que, en el año 2002, un 6\% de las encuestas realizadas por sus socios emplearon esa tecnología (Alós, 2003). En las siguientes ediciones no se hace ninguna referencia al tema, y es a partir del año 2005 cuando periódicamente se informa del número de dispositivos CAPI. En el año 2005 había, en las empresas españolas asociadas a AEDEMO, alrededor de 1.800 dispositivos CAPI, que, considerando que ese año se realizaron aproximadamente 3,276 millones de encuestas presenciales (Díaz de Rada y Portilla, 2015), supone alrededor de 1.820 encuestas por dispositivo; cifra que desciende a 717 en el año 2010. El análisis de la evolución de los dispositivos (tabla 1) revela un crecimiento sostenido durante todo el período, con aumentos notables en 2007 y 2006, y un leve repunte en los dos últimos años, lo que genera que el número de dispositivos se haya multiplicado por 2,5 en diez años ${ }^{2}$. Sin entrar en la consideración de identificar el momento en el

2. No deja de resultar sorprendente este aumento en los dos últimos años, mucho más cuando desde el año 2014 se realizan en España más encuestas autoadministradas que presenciales (AEDEMO, 2015 y 2016). 
Tabla 1. Número de dispositivos CAPI en las empresas españolas

\begin{tabular}{lrrrrrrrrrr}
\hline & 2005 & 2006 & 2007 & 2008 & 2009 & 2010 & 2011 & 2012 & 2014 & 2015 \\
\hline Número de dispositivos CAPI & 1.794 & 2.110 & 2.900 & 3.187 & 3.249 & 3.468 & 3.638 & 3.711 & 4.030 & 4.433 \\
Incremento porcentual anual & & $17,6 \%$ & $37,4 \%$ & $9,9 \%$ & $1,9 \%$ & $6,7 \%$ & $4,9 \%$ & $2,0 \%$ & $7,4 \%$ & $10,3 \%$ \\
\hline
\end{tabular}

Fuente: AEDEMO, varios años.

cual España tiene «suficientes» dispositivos de este tipo, el crecimiento experimentado en los últimos tiempos desvela que, en el año 2011 —momento de realización de la investigación empleada en este artículo-, la mayor parte de las encuestas presenciales que tenían lugar en España se hacían con "papel y lápiz».

¿Qué factores pueden explicar esta situación? ¿Puede aludirse a la notable inversión en equipos o más bien al hecho de que el empleo del CAPI no genera los avances localizados en otros contextos? En esta línea, una exhaustiva búsqueda bibliográfica sobre el tema en nuestro idioma localiza una ausencia de investigaciones acerca de dicha cuestión, a excepción de los trabajos de Lamas (2002, 2003) con el Estudio General de Medios.

Un segundo factor que justifica la realización de este trabajo es el hecho de que las primeras aplicaciones del CAPI, como se ha señalado, se realizaron con cuestionarios sobre comportamientos, y esta temática ha predominado en la mayor parte de investigaciones sobre sus ventajas y desventajas. De hecho, la práctica totalidad de los hallazgos señalados en las páginas anteriores se han realizado en cuestionarios sobre comportamientos, y apenas existen estudios con cuestionarios sobre actitudes. Considerando que esta situación supone una importante carencia en las encuestas sobre el tema, ello unido a la ausencia de investigaciones en España, se plantea el presente trabajo, cuya hipótesis es que existen diferencias sustanciales en la calidad de respuesta al cuestionario según el modo de administración (papel tradicional o electrónico). Del gran número de indicadores de calidad existentes, este trabajo centra su atención en la cantidad de respuestas «no sustanciales» en preguntas, esto es el número de "no sabe» $\mathrm{y}$ "no responde», así como la adecuación o inadecuación en seguir las instrucciones de los filtros de preguntas, dos de los aspectos más destacados en la literatura sobre el tema.

Una gran parte de las investigaciones publicadas sobre el tema se han centrado en comparar la no respuesta total, desvelando tasas similares en PAPI y CAPI (entre otros, West y Groves, 2013; Pforr et al., 2015; Lynn, 2016). Las que se han ocupado de la no respuesta parcial (no respuesta en cada pregunta) lo abordan sin considerar las diferencias según el tipo de preguntas ni el efecto producido por el lugar del cuestionario donde están colocadas. Tan solo la comparativa entre cerradas y abiertas ha generado alguna discusión, y se ha llegado a la conclusión de que no hay diferencia en el número de respuestas proporcionadas (Couper y Hansen, 2002). 
3. Diseño de la investigación: estudio postelectoral de las elecciones autonómicas y municipales (2011) en la Comunidad de Madrid (estudios CIS 2893 y 2894)

\subsection{Fuente de datos}

El universo es la población con derecho a voto en las elecciones autonómicas de la Comunidad de Madrid y que residía en esta comunidad entre el 27 de mayo y el 1 de julio del año 2011 (fechas de inicio y de finalización del trabajo de campo). La elección de los hogares donde se realizaron las entrevistas siguió el diseño habitual de los estudios llevados a cabo por el Centro de Investigaciones Sociológicas: diseño muestral en varias etapas en el que las unidades primarias (municipios) y las unidades secundarias (secciones censales) son elegidas de forma aleatoria proporcional, y las unidades últimas (individuos dentro de las viviendas) por rutas aleatorias y cuotas de sexo y edad.

Con la encuesta efectuada mediante papel y lápiz fueron respondidos 922 cuestionarios - de los 1.000 planificados- en 36 municipios entre el 27 de mayo y el 1 de julio (CIS, 2011a). Utilizando el ordenador se desarrollaron 455 entrevistas - de las 500 planificadas - en 24 municipios entre el 31 de mayo y el 20 de junio (CIS, 2011b). Es preciso indicar, tal y como se señala en la nota de investigación realizada sobre el estudio (CIS, 2012), que los mismos entrevistadores participaron en ambos estudios (p. 4) después de haber recibido formación específica de la aplicación informática. Estos trabajaron de forma exclusiva para este estudio durante los primeros cuatro días (p. 7) y las incidencias detectadas fueron de escasa relevancia (p. 12).

De dicha descripción se desprende que este diseño de investigación cumple las recomendaciones propuestas por Holbrook et al. (2003) para conocer los efectos de la modalidad de administración del cuestionario:

1. Un grupo debe ser entrevistado cara a cara, y un grupo diferente, mediante otra modalidad (con administración CAPI en el presente estudio). No debe entrevistarse a un mismo grupo utilizando ambas modalidades.

2. Ambas encuestas deben ser muestras representativas de un mismo universo.

3. Los entrevistados seleccionados mediante una modalidad deben ser entrevistados de esa forma. No es posible cambiar la modalidad de recogida de información con el fin de lograr la cooperación de las personas que muestran poca disposición a responder (o que han rechazado participar).

4. Deben emplearse entrevistas individuales con ambas modalidades.

5. Los entrevistados no deben tener capacidad decisoria para elegir cómo desean ser entrevistados, sino ser asignados por el diseño de investigación.

6. Los cuestionarios empleados deben ser idénticos (ver siguiente apartado).

\subsection{El instrumento de medida}

Se utilizó un cuestionario de 73 preguntas que fue respondido durante un promedio de 19 minutos en la encuesta con papel y lápiz. Casi una tercera 
parte del cuestionario (22 preguntas) recogen información sociodemográfica, y el resto (51 preguntas) son específicas de la temática de la investigación estudio postelectoral- («preguntas de contenido»). La mayoría de estas son de respuesta única (forced choice), presentan las categorías en formato vertical (formato abanico) y disponen de entre dos y nueve categorías de respuesta, aunque predominan las preguntas con cuatro y cinco. Hay también ocho preguntas de batería y seis preguntas de respuesta múltiple: dos dicotómicas, con seis y siete posibles respuestas, y el resto categóricas que limitan las respuestas a dos (8 variables). Además, el cuestionario cuenta con una escala de autodefinición de ideología política.

Las 95 variables respuestas proporcionadas por las preguntas «de contenido" (no sociodemográficas) se justifican porque un gran número de preguntas precisa de más de una respuesta (multirrespuestas y preguntas de batería ${ }^{3}$ ) y, por otro lado, por la no realización (salto) de determinadas cuestiones producida por la existencia de preguntas filtro. Es importante dedicar un espacio a este asunto por la simplificación de las tareas del entrevistador que supone el CAPI, especialmente cuando existen rutas complicadas. Este cuestionario dispone de ocho preguntas filtro (ver parte izquierda del cuadro 1) que condicionan la realización de quince preguntas contingentes (Alvira, 2011) o filtradas, tal y como se presenta esquemáticamente en el cuadro 1 . El texto literal de cada pregunta está disponible en la página web del CIS (2011a).

Los filtros son identificados en el cuestionario de papel con una flecha que indica el salto de una categoría a una determinada pregunta contingente. Son preguntas que aparecen sangradas en el cuestionario, suelen tener el nombre de la pregunta que las filtra con una letra $(a, b, c$, etc.) y están colocadas dentro de un recuadro para facilitar su identificación (Azofra, 1999). Cuando terminan, suele aparecer un recuadro - para el encuestador - con el texto «a todas las personas entrevistadas». La mayor parte de los filtros son sencillos y resulta fácil su identificación, pero hay tres situaciones relativamente complejas en los cuestionarios utilizados.

1. La primera está referida a la pregunta 19 , que condiciona la realización de seis preguntas (de la 19a a la 20b) en función de la respuesta obtenida. Así, por ejemplo, los que declaran que no pudieron votar (opción 1) deben «saltar» dos preguntas (preg. 19a, 19b), para ser preguntados por las razones que le impidieron votar (preg. 19c) y, posteriormente, saltar otras tres preguntas (sobre el voto) hasta la pregunta 21 (preg. 20, 20a y 20b).

Los que decidieron no votar saltan la pregunta de las razones que impiden votar (preg. 19c) y las tres preguntas sobre el voto.

3. En el apartado 3.c se analiza la influencia de las preguntas que producen más de una respuesta. Díaz de Rada (2015) ha llevado a cabo un análisis centrado en las preguntas de batería analizando el promedio de las no respondidas en cada modalidad; repetición de la misma respuesta en varios ítems de una pregunta y elección de las respuestas más fáciles (extremos de la respuesta y punto central). 
Cuadro 1. Preguntas filtro y preguntas filtradas o contingentes

\begin{tabular}{|c|c|c|c|c|}
\hline $\begin{array}{l}\text { Número } \\
\text { de pregunta } \\
\text { filtro }\end{array}$ & $\begin{array}{l}\text { Temática de la } \\
\text { pregunta filtro }\end{array}$ & $\begin{array}{l}\text { Temática de preguntas } \\
\text { contingentes (no proceden) }\end{array}$ & $\begin{array}{l}\text { Nombre de } \\
\text { preguntas no } \\
\text { respondidas }\end{array}$ & $\begin{array}{l}\quad \mathrm{N} .{ }^{\circ} \text { de } \\
\text { variables no } \\
\text { respondidas }\end{array}$ \\
\hline P13 & $\begin{array}{l}\text { Seguimiento de la campaña } \\
\text { electoral a través de Internet. }\end{array}$ & $\begin{array}{l}\text { Frecuencia seguimiento, tipo } \\
\text { páginas web. }\end{array}$ & preg. 13a y $13 b$ & 6 \\
\hline P17 & $\begin{array}{l}\text { Decisión de voto en las } \\
\text { elecciones autonómicas. }\end{array}$ & $\begin{array}{l}\text { Partidos entre los que dudó } \\
\text { (dos partidos). }\end{array}$ & preg. $17 a$ & 2 \\
\hline P18 & $\begin{array}{l}\text { Influencia en el voto de } \\
\text { las movilizaciones del 15M. }\end{array}$ & $\begin{array}{l}\text { Sentido en el que las ha } \\
\text { considerado. }\end{array}$ & preg. 18a & 1 \\
\hline \multirow[t]{6}{*}{ P19 } & $\begin{array}{l}\text { Participación electoral en las } \\
\text { últimas elecciones autonómicas: }\end{array}$ & & & \\
\hline & \multirow[t]{2}{*}{ No pudieron votar } & \multirow[b]{2}{*}{$\begin{array}{l}\text { Razón por la que no votó. } \\
\text { Momento de decisión de no } \\
\text { votar. } \\
\text { Momento decide el voto, } \\
\text { decisión (partido al que ha } \\
\text { votado), razón principal. }\end{array}$} & preg. 19a & 6 \\
\hline & & & $\begin{array}{l}\text { preg. } 19 \mathrm{~b} \\
\text { preg. } 20,20 \mathrm{a} \\
\text { y } 20 \mathrm{~b}\end{array}$ & \\
\hline & Decidieron no votar & $\begin{array}{l}\text { Razón por la que no pudo } \\
\text { votar. } \\
\text { Momento decide, decisión, } \\
\text { razón. }\end{array}$ & $\begin{array}{l}\text { preg. } 19 \mathrm{c} \\
\text { preg. } 20,20 \mathrm{a} \\
\text { y } 20 \mathrm{~b}\left(^{\star}\right)\end{array}$ & 4 \\
\hline & Votaron & $\begin{array}{l}\text { Relacionadas con el } \\
\text { «no voto». }\end{array}$ & $\begin{array}{l}\text { preg. } 19 a, 19 b \\
\text { y } 19 c\left(^{*}\right)\end{array}$ & 4 \\
\hline & No responde & $\begin{array}{l}\text { No preguntados en preg. } \\
19 a, 19 b, 20 a, 20 b\left(^{\star}\right) \text {. }\end{array}$ & & 7 \\
\hline P20a & $\begin{array}{l}\text { Voto últimas elecciones } \\
\text { autonómicas. }\end{array}$ & Razón de voto a ese partido. & preg. 20b & 1 \\
\hline P25 & $\begin{array}{l}\text { Recuerdo de voto últimas } \\
\text { generales. }\end{array}$ & Partido al que votó. & preg. 25a & 1 \\
\hline P32 & Participación últimas elecc. aut. & $\begin{array}{l}\text { Momento de decisión de } \\
\text { participar. } \\
\text { Partido al que votó. } \\
\text { Razón por la que votó a ese } \\
\text { partido. }\end{array}$ & $\begin{array}{l}\text { preg. 32a } \\
\text { preg. 32b } \\
\text { preg. 32c }\end{array}$ & 3 \\
\hline P36 & Participación electoral elec. aut. & Partido al que votó. & preg. $32 \mathrm{a}$ & 1 \\
\hline Total & 8 & 28 & & 24 \\
\hline
\end{tabular}

* No sumadas al haber sido consideradas anteriormente en la primera respuesta de la pregunta 19 (No pudieron votar) y primera opción de mla segunda (razón por la que decició no votar).

Fuente: elaboración propia con datos de los estudios 2893 y 2894 del CIS (año 2011). $N=1.377$.

Los que votaron saltan las tres preguntas sobre las razones de no votar. Los que no responden saltan las preguntas sobre las razones por las que no votaron y las tres sobre el voto (ver cuadro 1 ).

2. La segunda «situación compleja» es la existencia de un «doble filtro» en la pregunta 20, porque una pregunta filtrada (preg. 20a — partido al que votó en las elecciones autonómicas-) vuelve a realizar un segundo filtro (preg. 
$20 \mathrm{~b}$ - razones de votar a un determinado partido-) con una pregunta "no sangrada», colocada al mismo nivel de margen.

3. Algo parecido sucede en la pregunta 32, porque una pregunta filtrada (preg. $32 \mathrm{~b}$ - partido al que votó en las elecciones-) vuelve a realizar un segundo filtro (preg. 32c —razones de esta elección-).

Considerando que el cuestionario recoge información acerca de 95 variables, el número de respuestas oscilará entre 75 (mayor número de preguntas contingentes) y 92 (menor número). En el anexo 1 se muestra la temática de cada una de las preguntas, así como las preguntas filtro y las contingentes. Esta situación de saltos condicionados "complejos» en función de la respuesta es susceptible de producir muchos errores, por lo que — se plantea como hipótesis - existirán grandes diferencias entre la encuesta efectuada mediante papel y lápiz y la efectuada mediante el sistema CAPI, en la medida en que esta última realiza los saltos automáticamente, y prácticamente ni el entrevistado ni el entrevistador llega a percibirlos.

\subsection{Objetivos y técnicas de análisis utilizadas}

Para la medición de la calidad en la respuesta del cuestionario, se seguirán las aportaciones de las publicaciones más relevantes (entre otras, Holbrook et al., 2007; Heerwegh y Loosveldt, 2008; Heerwegh, 2009; Schaeffer et al., 2010; Saris et al., 2010; Stoop et al., 2010), que evalúan la respuesta con los siguientes indicadores:

a) Número de no respuestas en el cuestionario (considerando «no sabe»y «no contesta») y constatar si varía en función del instrumento empleado en la recogida (PAPI/CAPI) o del nivel educativo, consecuencia del efecto satisficing-complacencia (Krosnick et al., 2002).

b) Influencia del número de categorías en las preguntas de respuesta única.

c) Número de respuestas proporcionadas a las preguntas multirrespuesta.

d) Detectar el número de no respuestas en las partes finales del cuestionario, con el fin de localizar la presencia de un «efecto cansancio» diferente en función de la modalidad de administración.

No se consideran otros aspectos señalados por la literatura, como son el mayor número de respuestas a preguntas abiertas (McClamroch, 2011), al tratarse de un cuestionario con preguntas cerradas, y la mayor brevedad en responder el cuestionario (Watson y Wilkins, 2015; Böhme y Stohr, 2015), al no disponer de esta información.

Dichos indicadores de calidad proporcionan variables con métrica cuantitativa que precisarán de una comparación de los valores medios entre cada muestra, por lo que será utilizado un test de hipótesis - concretamente la diferencias de medias - para determinar si las desigualdades son significativas, tal y como se ha procedido en investigaciones similares (Schräpler et al., 
2010). Cuando se localice una diferencia se empleará la regresión múltiple, para conocer la influencia de las variables sociodemográficas y la modalidad empleada para administrar el cuestionario. Como términos independientes se consideran la modalidad del cuestionario (papel o electrónico), sexo, edad y nivel de estudios terminados, dentro de los rasgos sociodemográficos de los entrevistados. También la relación con la actividad, la situación profesional y el estatus socioeconómico — según la definición del CIS—, dentro de los rasgos socioeconómicos.

\section{Resultados}

El primer aspecto a estudiar es constatar que ambas muestras son equivalentes, ya que únicamente con muestras equivalentes podrá asegurarse que las diferencias obtenidas (entre ambas submuestras) estarán producidas por la modalidad utilizada en la recogida de información. El análisis de variables sociodemográficas — sexo, edad, tipo de familia y nivel de estudios terminados- no revela diferencias. De las variables socioeconómicas tan solo el estatus socioeconómico — según la definición del CIS — presenta alguna diferencia, producida fundamentalmente por el mayor número de entrevistas en papel realizadas en el estrato más alto (un 10\% más) y por el menor número llevado a cabo en las viejas clases medias (un 6\% menos). En el resto de categorías (nuevas clases medias, obreros cualificados y no cualificados), las distribuciones son muy similares, como puede apreciarse en el anexo 2 . Las variables referidas al comportamiento político (abstención electoral y recuerdo de voto en las elecciones autonómicas de mayo de 2007) presentan distribuciones idénticas en ambos grupos (ver anexo 2). A excepción del estatus socioeconómico, las muestras son similares.

La exposición de resultados se organiza en cuatro partes. En primer lugar, se considera el número total de no respuestas producido por cada modalidad de recogida de información, diferenciando entre las preguntas de "contenido" y las sociodemográficas; para continuar con el análisis del segundo tipo de preguntas con más presencia en el cuestionario, las de respuesta única, que presentan las categorías en formato vertical (o formato abanico). El siguiente apartado se dedica al estudio del número de respuestas proporcionadas por las preguntas de respuesta múltiple. El análisis de la no respuesta en las preguntas finales del cuestionario precede a las conclusiones.

\subsection{Análisis global del cuestionario. No respuesta parcial}

En la tabla 2 se muestra el número medio de no respuestas conseguido en cada tipo de preguntas, diferenciando entre las sociodemográficas y el resto. El análisis del número de no respuestas en las primeras (sociodemográficas) muestra una distribución de frecuencias (número de no respuestas) entre el 0 y el 7; en la que más de la mitad de la población (un 55\%) responde a todas las preguntas y un $30 \%$ deja únicamente una sin responder. Esto genera una no respuesta 
Tabla 2. Número de preguntas no respondidas en cada cuestionario

\begin{tabular}{|c|c|c|c|c|}
\hline \multicolumn{5}{|c|}{ Número medio de preguntas no respondidas } \\
\hline & PAPI & CAPI & $\begin{array}{l}\text { T de Student } \\
\text { PAPI-CAPI }\end{array}$ & Promedio \\
\hline Preguntas sociodemográficas & & & & 0,67 \\
\hline No responde & 0,630 & 0,420 & $4,618^{\star \star}$ & 0,56 \\
\hline No sabe & 0,110 & 0,100 & 0,544 & 0,11 \\
\hline Preguntas de contenido & & & & 5,96 \\
\hline No responde & 2,770 & 1,200 & $8,020^{\star \star}$ & 2,25 \\
\hline No sabe & 3,670 & 3,780 & 0,472 & 3,71 \\
\hline \multicolumn{5}{|l|}{ Preguntas contingentes } \\
\hline No responde & 0,335 & 0,281 & 1,272 & 0,317 \\
\hline No sabe & 0,179 & 0,165 & 0,490 & 0,174 \\
\hline \multicolumn{5}{|c|}{ Porcentaje de preguntas no respondidas respecto al total de preguntas del cuestionario } \\
\hline & PAPI & CAPI & & Promedio \\
\hline Preguntas sociodemográficas & & & & $3,7 \%$ \\
\hline No responde & $3,5 \%$ & $2,3 \%$ & & $3,1 \%$ \\
\hline No sabe & $0,6 \%$ & $0,5 \%$ & & $0,6 \%$ \\
\hline Resto de preguntas & & & & $7,8 \%$ \\
\hline No responde & $4,1 \%$ & $1,5 \%$ & & $3,2 \%$ \\
\hline No sabe & $4,6 \%$ & $4,6 \%$ & & $4,6 \%$ \\
\hline
\end{tabular}

** Diferencia significativa al 0,01.

Fuente: elaboración propia con datos de los estudios 2893 y 2894 del CIS (año 2011). $N=1.377$.

media de 0,67 , producida fundamentalmente por el deseo manifiesto de no responder $(0,56)$, comportamiento que es significativamente más frecuente en la entrevista con papel que cuando la entrevista se hace con ordenador $(0,63 \mathrm{y}$ 0,42 , respectivamente). Cuando estos valores medios se comparan con el total de preguntas sociodemográficas (parte inferior de la tabla 2), se obtiene que un $3,7 \%$ de las preguntas queda sin responder, de las cuales la mayor parte (un $3,1 \%$ ) tiene un origen en el deseo manifiesto de no responder, porcentaje que aumenta hasta el 3,5\% en la encuesta realizada sobre papel.

Fue señalado en el párrafo anterior que un 30\% de los entrevistados responde completamente al cuestionario excepto a una pregunta: el nivel de ingresos del hogar. Cuando no se considera esta pregunta, el promedio de no respuestas desciende hasta 0,23 . Esta reducción es aún mayor en el caso de las entrevistas con ordenador $(0,11)$, lo que implica que la falta de respuesta a esta pregunta ha sido superior en la entrevista realizada en papel que en la llevada a cabo con ordenador (un 33,5\% y un $28,6 \%$, respectivamente), en línea con los hallazgos realizados en otros contextos que dan cuenta de la adecuación del CAPI para la recogida de información sensible (Baker y Bradburn, 1992; De Leeuw y Nicholls II, 1996: 9; Waterton y Duffy, 1984).

El carácter comportamental de las preguntas sociodemográficas explica su escaso número de no respuestas, por lo que, a partir de este momento, la 
atención se centrará en el resto de preguntas del cuestionario, dedicadas fundamentalmente a aspectos actitudinales e ideológicos. En estas la ausencia de respuestas aumenta notablemente, con un número que oscila entre el $0 \mathrm{y}$ el 72. La cantidad de personas que responde todo el cuestionario no llega al $10 \%$ (un 9,6\%), y la mitad de los entrevistados deja sin responder entre una y cinco preguntas. Estas magnitudes proporcionan un promedio de casi seis preguntas sin responder (exactamente un 5,96; segunda parte de la tabla 2), un $7,8 \%$ de las preguntas del cuestionario. El motivo de no proporcionar respuesta a estas preguntas se debe fundamentalmente al desconocimiento («no sabe»). En la tabla 2 se muestran escasas diferencias en este comportamiento según la modalidad utilizada en la entrevista. Investigaciones realizadas en otros contextos (entre otros, Groves et al., 2009; Lynn y Purdon, 1994) localizan una mayor respuesta en el CAPI, que es interpretada aludiendo a que algunos entrevistados consideran que el ordenador requiere una respuesta, mientras que un entrevistador que utilice papel y lápiz puede dejar sin responder algunas cuestiones (Lynn y Purdon, 1994).

En estas preguntas se observa un escaso número de «no responde», que desciende significativamente en las entrevistas realizadas con CAPI. Aunque en ocasiones esta situación se explica por las señales sonoras que emiten los equipos cuando alguna pregunta queda sin responder (De Leeuw y Nicholls II, 1996), no es atribuible a este caso, porque los aparatos utilizados no disponían de esta funcionalidad, ya que se deseaba que los dos cuestionarios fueran similares (CIS, 2012).

Un análisis en detalle del comportamiento de los encuestadores en las preguntas filtro desvela que todas se han administrado correctamente en ambas modalidades, algo que puede deberse a la pericia de los encuestadores del CIS y la formación específica que han recibido para este estudio (CIS, 2012). Respecto a la presencia de respuestas «no sabe» o a la existencia de preguntas sin contestación, en la tercera parte de la tabla 2 puede observarse que los cuestionarios CAPI consiguen un menor número de no respuestas en preguntas contingentes, diferencia que no llega a ser significativa en ninguno de los dos aspectos considerados ${ }^{4}$.

Explicar las diferencias en la no respuesta teniendo en cuenta únicamente la modalidad empleada en la recogida implica una perspectiva notablemente reducida a la hora de abordar el problema, por lo que se ha procedido a evaluar la influencia de los rasgos sociodemográficos en la no respuesta, atendiendo por separado al número de respuestas «no responde» y «no sabe». Para ello, se han elaborado dos regresiones considerando el número de "no sabe» y "no responde» como términos dependientes y, como independientes, la modalidad empleada en la administración del cuestionario — sexo, edad, nivel máximo de estudios alcanzado, relación con la actividad, situación profesional y estatus socioeconómico- . Se busca controlar la influencia de los rasgos sociodemográficos de los entrevistados siguiendo una metodología empleada en otras

4. Más adelante se profundizará en esta diferencia. 
Tabla 3. Modelo de regresión de la influencia de variables sociodemográficas y modalidad de recogida en el número de preguntas no respondidas en una regresión binomial negativa

\begin{tabular}{|c|c|c|c|c|c|c|}
\hline & \multicolumn{3}{|c|}{ Modelo 1: «No responde» } & \multicolumn{3}{|c|}{ Modelo 2: «No sabe» } \\
\hline & Coeficientes & $\begin{array}{l}\text { Error } \\
\text { estándar }\end{array}$ & OR & Coeficientes & $\begin{array}{l}\text { Error } \\
\text { estándar }\end{array}$ & OR \\
\hline Intersección & $-0,270$ & 0,2601 & 0,764 & 0,983 & $0,1876^{\star \star}$ & 2,673 \\
\hline \multicolumn{7}{|l|}{ Modalidad de recogida } \\
\hline (Ref.: CAPI) & 0,879 & $0,0976^{\star \star}$ & 2,409 & $-0,014$ & 0,0581 & 0,986 \\
\hline \multicolumn{7}{|l|}{ Variables sociodemográficas } \\
\hline $\begin{array}{l}\text { Sexo: varones } \\
\quad \text { (Ref.: mujeres) }\end{array}$ & $-0,071$ & 0,0829 & 0,931 & $-0,226$ & $0,0624^{\star *}$ & 0,797 \\
\hline Edad & 0,006 & $0,0034^{\star}$ & 1,006 & 0,002 & 0,0025 & 1,002 \\
\hline \multicolumn{7}{|l|}{$\begin{array}{l}\text { Nivel de estudios } \\
\quad \text { (Ref.: superiores) }\end{array}$} \\
\hline Primarios y menos & 0,404 & $0,1512^{*}$ & 1,498 & 0,905 & $0,1152^{\star \star}$ & 2,471 \\
\hline Secundarios & 0,132 & 0,1198 & 1,141 & 0,395 & $0,0954^{\star \star}$ & 1,485 \\
\hline FP y equivalentes & $-0,156$ & 0,1492 & 0,856 & 0,353 & $0,1106^{\star \star}$ & 1,423 \\
\hline Univ. (tres años) & $-0,115$ & 0,1647 & 0,891 & 0,285 & $0,1205^{\star}$ & 1,33 \\
\hline \multicolumn{7}{|l|}{ Variables socioeconómicas } \\
\hline $\begin{array}{l}\text { Relación con la actividad } \\
\text { (Ref.: ama de casa) }\end{array}$ & & & & & . & \\
\hline 0cupados & 0,040 & 0,1448 & 1,040 & $-0,098$ & 0,1024 & 0,906 \\
\hline Parados & 0,166 & 0,1637 & 1,180 & 0,009 & 0,1170 & 1,009 \\
\hline Jubilados & $-0,189$ & 0,1488 & 0,828 & $-0,212$ & $0,1042^{*}$ & 0,809 \\
\hline Estudiantes & $-0,264$ & 0,2825 & 0,768 & $-0,081$ & 0,1886 & 0,922 \\
\hline Situación profesional & 0,024 & 0,1716 & 1,024 & 0,045 & 0,1280 & 1,046 \\
\hline \multicolumn{7}{|l|}{$\begin{array}{l}\text { Estatus socioeconómico } \\
\text { (Ref.: clase alta/media alta) }\end{array}$} \\
\hline Obreros no cualificados & 0,262 & 0,1448 & 1,300 & 0,115 & 0,1080 & 1,122 \\
\hline Obreros cualificados & 0,134 & 0,1290 & 1,143 & $-0,045$ & 0,0961 & 0,956 \\
\hline Viejas clases medias & $-0,021$ & 0,2154 & 0,979 & 0,022 & 0,1536 & 1,022 \\
\hline Nuevas clases medias & 0,066 & 0,1144 & 1,068 & $-0,069$ & 0,0857 & 0,933 \\
\hline Razón verosimilitudes $\left(\mathrm{G}^{2}{ }_{0-1}\right)$ & $157,295^{\star \star}$ & & & $187,663^{\star \star}$ & & \\
\hline Pseudo $R^{2}$ & 0,0310 & & & 0,0415 & & \\
\hline Número de casos & 1278 & & & 1278 & & \\
\hline
\end{tabular}

OR Razón de razones.

* Relación significativa al 0,05.

${ }^{\star *}$ Relación significativa al 0,01.

Fuente: elaboración propia con datos de los estudios 2893 y 2894 del CIS (año 2011). $N=1.377$.

investigaciones que comparan modalidades visuales y orales (entre otras, Messer y Dillman, 2011; Messer et al., 2012).

En la tabla 3 se presentan los modelos construidos para cada una de las modalidades de falta de respuesta. Comparando ambos modelos se observa 
que el modo de administración de la encuesta influye significativamente en la intensidad del «no responde», caso distinto de lo que sucede con el «no sabe». Con relación al modelo 1 , y en base a los coeficientes obtenidos, se observa que la intensidad con la que los entrevistados dejan preguntas sin responder aumenta un $41 \%$ cuando se utiliza un cuestionario de papel (PAPI). Además del modo de administración, también influye la edad, aunque con intensidad menor, indicando que el número de no respuestas aumenta un $0,6 \%$ con cada año cumplido. La escasa influencia del nivel de estudios pone en cuestión los planteamientos de la teoría de la complacencia (Survey Satisfacing), que explica la baja calidad del cuestionario considerando el nivel educativo de los entrevistados (Krosnick et al., 2002). La modalidad de recogida presenta más relevancia que el nivel de estudios de los entrevistados.

Respecto al modelo que estudia la intensidad de la respuesta «no sabe» (modelo 2), la modalidad de administración no presenta una influencia significativa. El modelo apunta que las características que influyen en el número de respuestas "no sabe» son el sexo del entrevistado y su nivel de estudios. Considerando el primero, es más probable que se produzca un número menor de respuestas «no sabe» en hombres que en mujeres. En el nivel de estudios, la intensidad con la que se recurre al «no sabe» es significativamente inferior en universitarios que en aquellos que poseen menos estudios (hasta secundarios). La interacción entre ambas variables no arroja resultados significativos.

El bajo poder explicativo de ambos modelos, en línea con lo logrado en investigadores similares (entre otros, Messer et al., 2012; Zhang y Conrad, 2014), indica el escaso efecto de las variables sociodemográficas en el número de preguntas no respondidas.

Resumiendo, baja no respuesta en variables sociodemográficas y notablemente superior en las preguntas de contenido específicas de la temática de la investigación, referidas a un estudio postelectoral autonómico. En estas últimas, la mayor parte de la no respuesta se produce por desconocimiento ( «no sabe») y no existe diferencia entre las modalidades. En los deseos manifiestos de no responder es donde se encuentran más diferencias entre el PAPI y el CAPI. El primero duplica los valores del segundo.

\subsection{Calidad en la respuesta de preguntas de respuesta única con categorías presentadas en formato vertical}

Tras el análisis de todo el cuestionario se centrará la atención a las 36 preguntas de respuesta única (forced choice) que presentan las categorías de contestación en sentido vertical (formato abanico). El número de categorías varía entre dos y nueve, aunque algo más de la mitad (21 preguntas) tienen cuatro y cinco opciones de respuesta (cuadro 2). Además, debe tenerse en cuenta que la mitad de estas preguntas han sido contestadas con ayuda de una tarjeta de respuesta que fue mostrada al entrevistado, tal y como puede verse en el cuadro 2.

En la tabla 4 se presenta el número medio de no respuestas diferenciando las declaraciones manifiestas de no responder y las respuestas "no sabe», 
Cuadro 2. Clasificación de las preguntas de respuesta única con categorías verticales, considerando el número de categorías y el empleo de tarjetas de respuesta en la entrevista

\begin{tabular}{lllr}
\hline Número de categorías & Con tarjeta & Sin tarjeta & Total \\
\hline Dos & Ninguna & P13 y P33 & 2 \\
Tres & Ninguna & P7 y P8 & 2 \\
Cuatro & P13a, P20, P22 y P32a & P10, P18, P21 y P23 & 8 \\
Cinco & P19, P19b, P25, P29, P30 y P32 & P1, P2, P3, P4, P6, P9 y P20a & 13 \\
Seis & P12 (3 respuestas) y P18a & P25a y P36a & 4 \\
Siete & P17, P20b y P32c & P32b & 4 \\
Ocho y más & P19c, P31 y P36 & & 3 \\
\hline Número total de preguntas: & 18 & 18 & 36 \\
\hline
\end{tabular}

Fuente: elaboración propia con datos de los estudios 2893 y 2894 del CIS (año 2011). $N=1.377$.

considerando el número de categorías y el empleo o no de tarjetas. Comenzando con las preguntas aplicadas con ayuda de tarjetas, la comparación de las columnas "promedio" vuelve a destacar la baja cifra de respuestas "no sabe», notablemente inferior al "no responde» en las preguntas con cuatro, cinco y seis categorías; situación que cambia en las preguntas con siete y más categorías. Este hecho puede estar ocasionado por la presencia de un efecto última opción generado por una administración "oral» en lugar de "visual» (utilizando tarjetas), puesto que los encuestadores han señalado que menos de la mitad de las entrevistas (exactamente un 45\%) emplearon todas las tarjetas de respuesta (Tourangeau et al., 2013).

La comparación entre modalidades únicamente desvela diferencias en el «no responde»: la aplicación mediante papel y lápiz presenta magnitudes de no respuesta más elevadas que la aplicación con CAPI, aunque solo tres presentan diferencias significativas: las que tienen cuatro, cinco y siete categorías. La diferencia en estas últimas pudiera estar causada por no administrar todas las tarjetas, aspecto que no es posible corroborar al no disponer de información sobre la correcta aplicación de tarjetas en la administración CAPI.

En las preguntas sin tarjetas, el número de «no sabe» es también inferior a la no respuesta, excepto en las preguntas con tres categorías (ver columnas "promedio»). La comparación entre administraciones desvela que únicamente hay diferencia en la no respuesta, y esta vuelve a ser menor en las preguntas realizadas con CAPI, aunque la diferencia solo es significativa en las preguntas con cinco y menos categorías de respuesta.

El análisis específico de las preguntas contingente muestra una ligera respuesta menor en el cuestionario CAPI (menor no respuesta), diferencia que únicamente llega a ser significativa en las preguntas filtro de cinco categorías; precisamente en la "ruta compleja» que mostraba la pregunta 9 y también en las «rutas semicomplejas» de la pregunta 20 y 32 . Este hallazgo supone dar «un paso más» a lo señalado en el apartado anterior, donde la consideración conjunta de las "faltas de respuesta» (excepto los no procede) de estas preguntas no presentaba diferencias según la modalidad. En este caso, 
Tabla 4. Promedio de no respuestas (diferenciando «no sabe» $y$ «no responde») en las preguntas con respuesta única con categorías verticales (comparación limitada a preguntas de contenido, sin considerar las sociodemográficas)

\begin{tabular}{|c|c|c|c|c|c|c|}
\hline \multicolumn{7}{|c|}{ Aplicadas con tarjeta (administración visual) } \\
\hline \multirow[b]{2}{*}{ Número de categorías } & \multicolumn{3}{|c|}{ No responde } & \multicolumn{3}{|c|}{ No sabe } \\
\hline & Promedio & PAPI & CAPI & Promedio & PAPI & CAPI \\
\hline Cuatro & 0,0813 & 0,104 & $0,035^{\star \star}$ & 0,0000 & 0,000 & 0,000 \\
\hline Cinco & 0,1351 & 0,173 & $0,057^{\star \star}$ & 0,1053 & 0,108 & 0,099 \\
\hline Seis & 0,0269 & 0,029 & 0,022 & 0,0000 & 0,000 & 0,000 \\
\hline Siete & 0,0363 & 0,048 & $0,043^{\star \star}$ & 0,0973 & 0,099 & 0,094 \\
\hline Ocho y más & 0,0668 & 0,074 & 0,053 & 0,1285 & 0,115 & 0,156 \\
\hline \multicolumn{7}{|c|}{ Aplicadas sin tarjeta (administración oral) } \\
\hline & \multicolumn{3}{|c|}{ No responde } & \multicolumn{3}{|c|}{ No sabe } \\
\hline Número de categorías & Promedio & PAPI & CAPI & Promedio & PAPI & CAPI \\
\hline Dos & 0,1206 & 0,132 & $0,097^{\star}$ & 0,0000 & 0,000 & 0,0000 \\
\hline Tres & 0,0203 & 0,028 & $0,004^{\star \star}$ & 0,2665 & 0,281 & 0,2370 \\
\hline Cuatro & 0,0399 & 0,049 & $0,022^{*}$ & 0,0218 & 0,019 & 0,0264 \\
\hline Cinco & 0,1503 & 0,168 & $0,114^{\star}$ & 0,0712 & 0,078 & 0,0570 \\
\hline Seis & 0,1830 & 0,182 & 0,185 & 0,0632 & 0,072 & 0,0462 \\
\hline Siete & 0,1009 & 0,102 & 0,099 & 0,0000 & 0,000 & 0,0000 \\
\hline \multicolumn{7}{|c|}{ Preguntas contingentes (considerando el número de categorías de las preguntas filtro) } \\
\hline & \multicolumn{3}{|c|}{ No responde } & \multicolumn{3}{|c|}{ No sabe/no recuerda } \\
\hline Número de categorías & Promedio & PAPI & CAPI & Promedio & PAPI & CAPI \\
\hline Dos & 0,0000 & 0,0000 & 0,0000 & 0,0000 & 0,0000 & 0,000 \\
\hline Cuatro & 0,0203 & 0,0203 & 0,0163 & 0,0501 & 0,0530 & 0,044 \\
\hline Cinco & 0,0741 & 0,0944 & $0,0330^{\star \star}$ & 0,1110 & 0,1190 & 0,107 \\
\hline Seis & 0,1830 & 0,1846 & 0,1822 & 0,0632 & 0,0716 & 0,046 \\
\hline Siete & 0,0428 & 0,0434 & 0,0400 & No ofrecida & & \\
\hline
\end{tabular}

* Diferencia significativa al 0,05 en el de promedio de respuestas «no sabe» $y$ «no responde».

** Diferencia significativa al 0,01 en promedio de respuestas «no sabe» $y$ «no responde».

Fuente: elaboración propia con datos de los estudios 2893 y 2894 del CIS (año 2011). $N=1.377$.

considerar las preguntas diferenciando el número de categorías produce una mejor respuesta (menor número de «no respuestas») en los cuestionarios respondidos con CAPI.

\subsection{Calidad en la respuesta en preguntas de respuesta múltiple}

En tercer lugar se presentan las preguntas con menos presencia en el estudio utilizado. El cuestionario cuenta con seis preguntas de respuesta múltiple, dos con respuestas sí/no sobre un conjunto de categorías (dicotómica) y cuatro donde se ofrece un conjunto de categorías y, tras responder, se le plantea al entrevistado si tiene alguna otra respuesta (categórica limitada a dos respuestas). Conviene tener en cuenta que, en la mayor parte de las preguntas - excepto 
en la pregunta 16 y la 17a (múltiple dicotómica y categórica respectivamente) - , los entrevistados contaban con una tarjeta donde se mostraban todas las categorías de respuesta.

El análisis de este tipo de preguntas se centrará específicamente en el número de respuestas conseguidas por cada modalidad, planteando como hipótesis que los entrevistados por ordenador proporcionan más. El análisis de la primera pregunta dicotómica, donde los entrevistados podrían dar hasta cinco respuestas, desvela un mayor número de respuestas en la modalidad CAPI, una diferencia baja pero significativa. En la pregunta siguiente (preg. 16), el entrevistado puede dar hasta siete respuestas y, aunque el CAPI consigue más respuestas, la diferencia no llega a ser significativa (tabla 5). Es posible que la no utilización de tarjetas de respuesta pueda explicar las diferencias con la pregunta $13 \mathrm{~b}$.

De las preguntas multirrespuesta categórica tan solo dos — de cuatropresentan una diferencia significativa según la modalidad de entrevista. En los partidos entre los que dudó a la hora de votar (preg. 17a), los entrevistados con CAPI o no responden o dan dos respuestas y desciende notablemente el número de entrevistados que proporcionan —únicamente- una respuesta. En la pregunta sobre las razones por las que no votó en las últimas elecciones autonómicas (preg. 19a), el cuestionario tradicional recoge más respuestas, quizás influenciado por la utilización de una tarjeta con las respuestas, que no fue empleada en la pregunta 17a. En el resto de aspectos, las diferencias son escasas y no significativas. En suma, resultados no muy concluyentes en las preguntas multirrespuesta categórica.

\subsection{Influencia del "efecto cansancio» en cada modalidad y reducción de los efectos de deseabilidad social}

El último de los aspectos señalados en el apartado 2.c era detectar el número de no respuestas en las partes finales del cuestionario con el fin de localizar la presencia de un "efecto cansancio" que pudiera ser diferente según la modalidad de administración utilizada. Para llevar a cabo este aspecto, el cuestionario fue dividido en cuatro partes 5 (de aproximadamente 24 variables cada una), con el fin de comparar la última y la penúltima parte con las dos primeras. Un análisis del cuestionario no desvela grandes diferencias entre el número de categorías en las preguntas de cada parte.

Con el fin de comparar el mismo número de preguntas, el promedio de no respuestas en cada parte fue dividido entre el número de preguntas, con lo que se obtuvieron valores comparables. Considerando que el objetivo es conocer hasta qué punto el cansancio produce un mayor número de no respuestas, en la tabla 6 se muestra el número promedio de «no responde» de la primera mitad, el tercer cuarto y el último cuarto.

5. Considerando únicamente las preguntas de contenido, excluyendo las preguntas sociodemográficas por su menor respuesta, tal y como se ha señalado en el apartado 4.a. 
Tabla 5. Número de respuestas proporcionado por las preguntas de respuesta múltiple (porcentajes de columna)

\begin{tabular}{|c|c|c|c|c|}
\hline \multicolumn{5}{|c|}{ Multirrespuesta dicotómica (media de respuestas) } \\
\hline & PAPI & CAPI & $\begin{array}{l}\text { T de Student } \\
\text { PAPI-CAPI }\end{array}$ & $\begin{array}{l}\text { Promedio } \\
\text { no respuest. }\end{array}$ \\
\hline $\begin{array}{l}\text { Preg. 13b: Seguimiento de la campaña mediante } \\
\text { Internet** Media de respuestas }\end{array}$ & 1,618 & 1,81 & $2,03^{\star}$ & 1,704 \\
\hline $\begin{array}{l}\text { Preg. 16: Forma de seguimiento de la campaña } \\
\text { electoral: vio por televisión propaganda electoral, } \\
\text { leyó folletos, cartas, etc. Media de respuestas }\end{array}$ & 2,292 & 2,41 & 1,41 & 2,330 \\
\hline \multicolumn{5}{|c|}{$\begin{array}{cc}\text { Multirrespuesta categórica (porcentajes verticales) } \\
\end{array}$} \\
\hline & PAPI & CAPI & $\begin{array}{l}\text { Chi cuadrado } \\
\text { PAPI-CAPI }\end{array}$ & Promedio \\
\hline $\begin{array}{l}\text { Preg. 17a: Partidos entre los que dudó en } \\
\text { las elecciones autonómicas del año } 2011^{\star \star \star}\end{array}$ & $7,726^{\star}$ & & & \\
\hline Ninguna respuesta & $6,0 \%$ & $11,7 \%$ & & $7,7 \%$ \\
\hline Una respuesta & $9,8 \%$ & $1,3 \%$ & & $7,3 \%$ \\
\hline Dos respuestas & $84,2 \%$ & $87,0 \%$ & & $85,1 \%$ \\
\hline$N$ & 184 & 77 & & 261 \\
\hline $\begin{array}{l}\text { Preg. 19a: Razones por las que no votó } \\
\text { en las últimas elecciones autonómicas }{ }^{\star \star \star \star}\end{array}$ & 5,960 & & & \\
\hline Una respuesta & $11,1 \%$ & $23,4 \%$ & & $15,4 \%$ \\
\hline Dos respuestas & $86,8 \%$ & $74,0 \%$ & $5,909^{*}$ & $82,4 \%$ \\
\hline$N$ & 144 & 77 & & 221 \\
\hline \multicolumn{5}{|l|}{$\begin{array}{l}\text { Preg. 24: Temas más importantes para el nuevo } \\
\text { gobierno de la comunidad }\end{array}$} \\
\hline Ninguna respuesta & $2,1 \%$ & $0,7 \%$ & & $1,6 \%$ \\
\hline Una respuesta & $3,5 \%$ & $3,5 \%$ & & $3,5 \%$ \\
\hline Dos respuestas & $94,5 \%$ & $95,8 \%$ & & $94,9 \%$ \\
\hline$N$ & 922 & 455 & & 1.377 \\
\hline \multicolumn{5}{|l|}{$\begin{array}{l}\text { Preg. 31a: Autodefinición política: conservador, } \\
\text { democristiano, liberal, etc. }\end{array}$} \\
\hline Ninguna respuesta & $13,4 \%$ & $15,6 \%$ & & $14,2 \%$ \\
\hline Una respuesta & $49,1 \%$ & $45,5 \%$ & & $47,9 \%$ \\
\hline Dos respuestas & $37,4 \%$ & $38,9 \%$ & & $37,9 \%$ \\
\hline$N$ & 922 & 455 & & 1.377 \\
\hline
\end{tabular}

* Significación al 0,05.

** Respondida únicamente por los 385 entrevistados (un $28 \%$ de la muestra) que siguió la campaña mediante Internet.

*** Respondida únicamente por los que dudaron entre varios partidos, entre un partido y abstenerse, y entre un partido y votar en blanco, un $19 \%$ de la muestra (261 entrevistados).

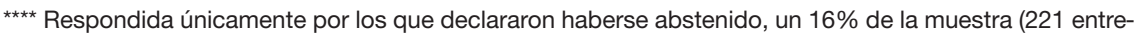
vistados).

Fuente: elaboración propia con datos de los estudios 2893 y 2894 del CIS (año 2011). N = 1.377. 
En el número de «no responde» se aprecia un aumento progresivo a medida que aumenta el cuestionario: de un promedio de 0,0068 preguntas sin responder en la primera mitad a 0,067 en la última parte del cuestionario. Considerando las modalidades, el aumento a medida que transcurre la entrevista es muy superior en el caso del cuestionario tradicional que en el CAPI. Destaca el bajo número de no respuestas del CAPI en la primera mitad.

No se realiza el análisis con las preguntas «no sabe» por las diferentes temáticas abordadas en cada parte del cuestionario, lo que dificulta notablemente la comparación. Así, la primera mitad se ocupa de la valoración del gobierno de la comunidad y del seguimiento de la campaña electoral, mientras que las otras dos están referidas a la votación en los diferentes comicios, valoración de líderes e identificación de los problemas más relevantes.

\section{Conclusiones y discusión}

La correcta utilización de la encuesta precisa conocer en detalle sus límites y potencialidades (Sánchez Carrión, 2012), motivo por el cual se ha planteado este trabajo que compara la calidad de las respuestas obtenidas mediante un cuestionario que es administrado en papel y con ordenador en dos muestras equivalentes. La medición de la calidad se ha realizado considerando el promedio de preguntas no respondidas en cada modalidad (diferenciando entre «no sabe» $\mathrm{y}$ «no responde»), la ausencia de respuesta en preguntas de respuesta única y el número de respuestas logrado por las preguntas de respuesta múltiple, así como las no respuestas en las preguntas finales del cuestionario.

El análisis del cuestionario al completo desvela una alta respuesta en las preguntas sociodemográficas —el 30\% deja sin responder una pregunta y un $15 \%$ más de una-, pero cuando esta tiene lugar, está generada — fundamentalmente- por un deseo de no responder. El promedio de preguntas no respondidas es 0,67 - un 3,7\% del cuestionario-y las preguntas peor respondidas fueron: persona que aporta más ingresos al hogar (65 entrevistados, un $6,1 \%$ ), creencia religiosa (52 entrevistados, un 3,8\%) y autodefinición de clase social (43 entrevistados, un 3,1\% de la muestra). En todas ellas la no respuesta es significativamente mayor en el cuestionario PAPI.

En el resto de preguntas del cuestionario, centradas fundamentalmente en aspectos actitudinales e ideológicos, se produce un gran aumento de la falta de respuesta, con un promedio de (casi) seis preguntas sin responder. El motivo de no proporcionar respuesta en estas preguntas se produce fundamentalmente por desconocimiento («no sabe»), cifra que no varía en función de la modalidad de recogida. Solo el rechazo a responder («no responde») es significativamente distinto cuando se comparan el sistema PAPI y el CAPI, siendo menor en el segundo. El análisis de regresión ha desvelado que la modalidad de recogida y - en menor medida - la edad son las únicas variables que presentan relación con el número de «no respuestas». En las respuestas «no sabe» el nivel de estudios es la variable con más influencia. En ellas, las personas con menos estudios presentan un mayor número de no respuestas debido al desconocimiento. 
Tabla 6. Número de preguntas no respondidas en cada parte del cuestionario

\begin{tabular}{lcccc}
\hline \multicolumn{5}{c}{ Número medio de «no responde» por pregunta } \\
\hline Preguntas de contenido & PAPI & CAPI & T de Student PAPI-CAPI & Promedio \\
\hline Primera mitad & 0,0094 & 0,0014 & $4,906^{\star \star}$ & 0,0068 \\
Tercer cuarto & 0,0221 & 0,0147 & $3,117^{\star \star}$ & 0,0196 \\
Cuarta parte & 0,0779 & 0,0340 & $9,856^{\star \star}$ & 0,0674 \\
\hline
\end{tabular}

* Diferencia significativa al 0,05.

** Diferencia significativa al 0,01.

Fuente: elaboración propia con datos de los estudios 2893 y 2894 del CIS (año 2011). $N=1.377$.

No hay diferencia entre las modalidades a la hora de aplicar correctamente las preguntas filtro, y la comparación de las no respuestas en las preguntas contingentes desvela un ligero menor número de no respuestas en los cuestionarios CAPI, diferencia que no llega a ser significativa.

El análisis de las 36 preguntas de respuesta única con categorías dispuestas en sentido vertical desvela que la falta de respuesta se caracteriza por elevadas elecciones de «no responde». Son preguntas que presentan entre tres y nueve categorías, si bien más de la mitad tienen cuatro y cinco. La encuestas de papel y lápiz presentan más no respuestas que las realizadas con CAPI, aunque solo tres muestran diferencias significativas: cuatro, cinco y siete categorías en las preguntas con tarjeta, y tres, cuatro y cinco en las que no tienen tarjeta.

En las preguntas de respuesta múltiple el análisis se centró en el número de respuestas proporcionado por cada modalidad, considerando que los entrevistados por ordenador proporcionarían más respuestas. De las seis preguntas multirrespuesta solo tres presentan diferencias significativas, una dicotómica y dos categóricas. En la pregunta multirrespuesta dicotómica el CAPI proporciona más respuestas, pero en las dos multirrespuestas categóricas no se ofrecen resultados consistentes.

Se ha constatado también un aumento de preguntas no respondidas a medida que aumenta el tiempo de duración de la entrevista y el cuestionario tradicional consigue el doble de no respuestas que el cuestionario CAPI.

Es importante considerar que la literatura muestra un gran acuerdo en que el cambio en la modalidad tiene mayores efectos con cuestionarios complicados y con grandes tamaños muestrales. Consideramos que el presente cuestionario no cumple ninguna de estas premisas, al haberse respondido — en la modalidad tradicional — en 19 minutos y, además, porque tan solo dispone de 9 preguntas filtro, y de estas únicamente 1 presenta una «ruta compleja». Esto puede explicar la gran similitud entre las modalidades. El hecho de que el cuestionario CAPI no informe sobre el tiempo necesario para responderlo, ni que disponga de las incidencias habituales en los estudios del CIS, deja el análisis incompleto, al no proporcionar información del tiempo necesario de respuesta - menor en la totalidad de literatura analizada - y el número de intentos realizados para hacer la entrevista.

Sin desdeñar la importancia de la información incluida en los estudios 2893 y 2894 del Centro de Investigaciones Sociológicas, hay varios aspectos que, 
desgraciadamente, no pueden analizarse y que se quedan a la espera de que otros investigadores lo hagan. Considérense, entre otros, las mejoras en la gestión del trabajo de campo (AIMC, 2002; Lamas, 2002, 2003), la reacción de los entrevistados, en especial respecto a la satisfacción con la entrevista (Lamas, 2003) y las mayores posibilidades de ser reentrevistados, así como la mejora en la colaboración de colectivos difíciles (Lamas, 2002). Otro ámbito de interés es considerar hasta qué punto el cuestionario administrado mediante ordenador está menos afectado por efectos de respuesta como primacía, recencia, satisficing, etc., así como los posibles efectos con determinadas temáticas, como los temas sensibles. Dichos aspectos han sido investigados en otros contextos y han demostrado la mejora del CAPI frente al tradicional cuestionario de papel y lápiz. No menos importante es el (posible) ahorro de tiempo que suponen las entrevistas efectuadas con CAPI y el asunto del coste, fundamentalmente en el aumento de costes fijos (compra de equipos) y la necesidad de personal más formado para introducir el cuestionario en los equipos.

Algunos de estos aspectos han sido analizados por el Centro de Investigaciones Sociológicas y, aunque las incidencias de campo acontecidas en el cuestionario CAPI no están disponibles, hay una interpretación de estos en el trabajo Estudio piloto: Implantación del CAPI. Postelectoral elecciones autonómicas y municipales 2011 de la Comunidad de Madrid, que puede ser descargado directamente de la página web del CIS (http://www.cis.es/cis/opencms/ES/12_ NotasInvestigacion/Investigaciones/2012/NotaInvestigacion0007.html).

Sintetizando, la mayor parte de la "no respuesta» en las preguntas de opiniones y actitudes se ha producido por «no sabe», y son respuestas que apenas varía en función de la modalidad de administración utilizada, sino que están relacionadas con el nivel de estudios y el sexo del entrevistado. El número de respuestas «no sabe» desciende en los entrevistados con estudios más elevados y en los varones. La decisión de no responder es ligeramente inferior (un 2,2\%) y presenta diferencias según la modalidad utilizada, siendo siempre superior en las encuestas de papel y lápiz. El análisis en detalle considerando el tipo de preguntas no presenta grandes variaciones con esta tendencia general, ni aún cuando se analizan las tres preguntas filtro que presentan filtros más complejos.

\section{Referencias bibliográficas}

Alós, J. (2003). «Industria de los estudios de mercado en España 2002: Crecimos un 9\%». Investigación y Marketing, 80, 76-78.

Alvira, Francisco (2011). La encuesta: Una perspectiva general metodológica. Madrid: CIS. Cuadernos Metodológicos, 35.

Asociación Española de Estudios de Mercado, Marketing y Opinión (AEDEMO) (2015). Estudio de la industria de los estudios de mercado. Recuperado el 1 de julio de 2015, de <http://www.aedemo.es/aedemo/index.php?option=com_conte nt\&task=blogcategory\&id=37\&Itemid $=207>$.

- (2016). El sector de la investigación de mercados en España 2015. Recuperado el 15 de noviembre de 2016, de <http://www.aneimo.com/datosmercado.php>. 
Asociación para la Investigación de los Medios de Comunicación (AIMC) (2002). "El EGM de PAPI a CAPI». Linea Abierta (octubre).

Azofra, María José (1999). Cuestionarios. Madrid: Centro de Investigaciones Sociológicas. Cuadernos Metodológicos, 26.

BAKer, Reginald P. y Bradburn, Norman M. (1992). CAPI: Impacts on data quality and survey cost. Information Technology in Survey Research Discussion paper 10 .

BAKER, Reginald; BradBurn, Norman M. y JoHnSON, Robert A. (1995). «Computer assisted personal interviewing: An experimental evaluation of data quality». Journal of Official Statistics, 11 (4), 413-431.

Bergman, Lars R.; Kristiansson, Karl-Erik; Olofsson, Anita y SÄfström, Margaretha (1994). «Decentralised CATI versus paper and pencil interviewing: Effects on the results in the swedish labour force surveys». Journal of Official Statistics, 10 (2), 181-195.

BöHme, Marcus y STOHR, Tobias (2015). «Household Interview Duration Analysis in CAPI Survey Management». Field Methods, 26 (4), 390-405. <https://doi.org/10.1177/1525822X14528450>

Bourque, Linda B. y Clark, Virginia A. (1992). «Processing Data: The Survey Example». Paper Series on Quantitative Applications in the Social Sciences 07-085. Newbury Park, CA: Sage University.

Centro de Investigaciones Sociológicas (2002). Nuevas lineas de Investigación Social Aplicada para el estudio de la sociedad española. Encuentros CIS, Ávila, 2001. Madrid: Centro de Investigaciones Sociológicas.

- (2011a). Postelectoral elecciones autonómicas y municipales 2011: Comunidad de Madrid. Piloto CAPI, estudio CIS 2894. Recuperado el 14 de abril de 2013, de <http://www.cis.es/cis/opencm/ES/1_encuestas/estudios/resBusqueda.jsp?nEstudi oMin=2893\&nEstudioMax=2894\&mesIni=\&anioIni $=\&$ mesFin $=\& a n i o F i n=\& m$ uestraIni $=\&$ muestraFin $=\&$ publicado $=3 \&$ cualitativo $=3 \&$ ptitulo $=\&$ pcontenido $=\&$ ambito $=0 \&$ universo $=0 \&$ sexo $=0 \&$ edad $=0 \&$ btnBuscarE $=$ Buscar $>$.

- (2011b). Postelectoral elecciones autonómicas y municipales 2011. Comunidad de Madrid. Estudio CIS 2893.

- (2012). Informe breve estudio piloto implantación CAPI: Postelectoral elecciones autonómicas y municipales 2011. Comunidad de Madrid. Recuperado el 1 de enero de 2013, de <http://www.cis.es/cis/opencms/ES/12_NotasInvestigacion/Investigaciones/2012/NotaInvestigacion0007.html (en línea)>.

Couper, Mick P. y Hansen, Sue Ellen (2002). «Computer assisted interviewing». En: Gubrium, Jaber F. y HolsteIn, James A. (eds.). Handbook of Interview Research: Context and Methods. Thousand Oaks, CA: Sage.

DE LEEUW, Edith (2004). New technologies in data collection, questionnaire design and quality. San Sebastián: Instituto Vasco de Estadística / Eustat.

De LeEuW, Edith y Collins, Martin (1997). «Data collections methods and survey quality: An overview». En: LyberG, Lars E.; Biemer, Paul; Collins, Martin; DE Leeuw, Edith D.; Dippo, Carthyn; Schwarz, Norbert y Trewin, Dennis (eds.). Survey Measurement and Process Quality. Nueva York: Wiley.

De LEEuw, Edith D.; Hox, Joop J. y SNIJKERS, Ger (1995). «The effect of computerassisted interviewing on data quality: A review». International Journal of Market Research, 37, 325-344.

De LeEuW, Edith y Nicholls II, William (1996). «Technological innovations in data collection: Acceptance, data quality and cost». Sociological Research Online, 
1 (4). Recuperado el 13 de abril de 1999, de <http://www.socresonline.org.uk/ index_by_issue.html>. $<$ https://doi.org/10.5153/sro.50>

DíAZ DE RADA, Vidal (2015). «Calidad de los datos de preguntas de batería en encuestas presenciales: Una comparación de un estudio con cuestionario en papel y en formato electrónico». Revista Española de Investigaciones Sociológicas, 152, 167-178. <https://doi.org/10.5477/cis/reis.152.167>

DíAz DE RADA, Vidal y PORTILla, Idoia (2015). «Encuestas telefónicas: Estrategias para mejorar la colaboración». Perspectiva Empresarial, 2 (1), 97-115. <https://doi.org/10.16967\%2Frpe.v2n1a7>

Dielman, Lynn y Couper, Mick P. (1995). «Data quality in a CAPI survey: Keying errors». Journal of Official Statistics, 11 (2), 141-146.

Groves, Robert M. et al. (2009). Survey Methodology. Nueva York: Wiley.

HEERWEGH, Dirk (2009). «Mode differences between face-to-face and web surveys: An experimental investigation of data quality and social desirability effects». International Journal of Public Opinion Research, 21, 111-120. $<$ https://doi.org/10.1093/ijpor/edn054>

HeERweGH, Dirk y LoOSveldT, Geert (2008). «Face to face versus web surveying in a high internet coverage population». Public Opinion Quarterly, 72 (5), 836-846. <https://doi.org/10.1093/poq/nfn045>

Hernández Moreno, Antonio; Muñoz Conde, María y Bohórquez Baña, Rosa (2011). «Procesos de validación, depuración e imputación en las encuestas a hogares del Instituto Estadístico de Andalucía». Metodología de Encuestas, 13, 55-70.

Holbrook, Allyson L.; Green, Melanie C. y Krosnick, Jon A. (2003). «Telephone versus face-to-face interviewing of national probability samples with long questionnaires». Public Opinion Quarterly, 67, 79-125.

$<$ https://doi.org/10.1086/346010>

Holbrook, Alyson L.; Krosnick, Jon A.; Moore, David y Tourangeau, Robert (2007). «Response order effects in dichotomous categorical questions presented orally: The impact of question and respondent attributes». Public Opinion Quarterly, 71, 325-348. <https://doi.org/10.1093/poq/nfm024>

KenNEDY, Jean More.; LENGACHER, E. y DEMERATH, L. (1990). «Interviewer entry errors in CATI interviews». International conference on measurement errors in surveys. Arizona: Tucson.

Krosnick, J.A.; HolbroOK, A.L.; Berent, M.K.; Carson, R.T.; Hanemann, W.M.; Kopp, R.J.; Mitchell, R.C.; Presser, S.; Ruud, P.A.; Smith, V.K.; Moody, W.R.; Green, M.C. y Conaway, M. (2002). "The Impact of "No Opinion" Response Options on Data Quality: Non-Attitude Reduction or an Invitation to Satisfice?». Public Opinion Quarterly, 66 (3), 371-403. <https://doi.org/10.1086/341394>

LAMAS, Carlos (2002). Las entrevistas personales con ordenador: La experiencia del EGM. II Congreso de Investigación mediante Encuestas. Santiago de Compostela, 26 de septiembre.

- (2003). The EGM conversion to CAPI was completed. 2003 EMRO Conference. Kuopio, Finlandia, mayo.

LEPKOWSKI, James M. et al. (1995). «Exploring mode differences in interviewer entry errors». Proceedings of the Joint Statistical Meeting of the American Statistical Association, section on Survey Research Methods. Alexandría, VA: American Statistical Association. 
LYNN, Peter (2016). «Targeted Appeals for Participation in Letters to Panel Survey Members». Public Opinion Quarterly, 80 (3), 771-782. $<$ https://doi.org/10.1093/poq/nfw024>

LYNN, Peter y Purdon, Susan (1994). "Time series and lap-tops: The change to computer assisted interviewing». En: Jowell, Rogert (ed.). The British Social Attitudes. Aldershot: Dartmouth, 141-155.

MARTIN, Jean y MANNERS, Tony (1995). "Computer assisted personal interviewing in survey research». En: LEE, Raymond M. (ed.). Information technology for the social sciences. Londres: UCL Press.

McClamroch, Kristi J. (2011). «Evaluating the Usability of Personal Digital Assistants to Collect Behavioral Data on Adolescents with Paradata. Field Methods, 23 (3), 219-242. $<$ https://doi.org/10.1177/1525822X11405826>

Messer, Benjamin L.; Edwards, Michelle L. y Dillman, Don A. (2012). «Determinants of Item Nonresponse to Web and Mail Respondents in Three Address-Bases Mixed-Mode Surveys of the General Public». Survey Practice, 5 (2). Recuperado el 25 de mayo de 2015, de <http:/www.surveypractice.org/index.php/SurveyPractice/article/view/45/pdf>.

Messer, B.L. y Dillman, D.A. (2011). "Surveying the general public over the internet using address-based sampling and mail contact procedures». Public Opinion Quarterly, 75, 429-457. <https://doi.org/10.1093/poq/nfr021>

OlsEN, Randall J. (1992). The Effects of Computer-Assisted Interviewing on Data Quality. Colchester: Universidad de Essex.

Pforr, Klaus; Blohm, Michael; Blom, Annelies G. et al. (2015). «Are Incentive Effects on Response Rates and Nonresponse Bias in Large-scale, Face-to-face Surveys Generalizable to Germany? Evidence from Ten Experiments». Public Opinion Quarterly, 79 (3), 740-768. $<$ https://doi.org/10.1093/poq/nfv014>

Roth, Philip L. (1994). «Missing Data: A Conceptual Review for Applied Psychologists». Personal Psychology, 47, 537-560. <https://doi.org/10.1111/j.1744-6570.1994.tb01736.x>

SÁNCHEZ CARrión, Juan Javier (2012). "La encuesta, herramienta cognitiva». Papers, 97 (1), 169-192.

SARIS, Willem (1991). Computer Assisted Interviewing. Newbury Park, CA: Sage. Quantitative Research Methods Series, 63.

SARIS, Willem; ReVILla, Melanie y KrosnicK, Jon A. (2010). "Comparing questions with agree/disagree response options to question with item-specific response options». Survey Research Methods, 4 (1), 61-79 <https://doi.org/10.18148/srm/2010.v4i1.2682>

Schaeffer, Nora Cate; DyKema, Jeniffer y MAYNARD, Douglas W. (2010). «Interviewers and interviewing». En: MARSDEN, Peter V. y Wright, James D. (eds.). Handbook of Survey Research. 2. ${ }^{\mathrm{a}}$ ed. Bingley (Reino Unido): Emerald Group Publishing Limited.

SCHrÄPleR, J.P.; SCHUPP, J. y WAGNER, G.G. (2010). «Changing from PAPI to CAPI: Introducing CAPI in a longitudinal study». Journal of Official Statistics, 26 (2), 239-269.

STOOP, Inkele; Billiet, Jaak; KocH, Achim y FitzGerald, Rory (2010): Improving survey response: Lessons learned from the European Social Survey. Nueva York: Wiley. 
Tabachnick, Barbara G. y Fidell, Linda S. (1989). Using Multivariate Statistics. Nueva York: Harper \& Publishers.

Tourangeau, Roger; Conrad, Frederic G. y Couper, Mick P. (2013). The Science of Web Surveys. Nueva York: Oxford University Press.

Van Bastelaer, Alois; KerssemaKers, Frans y SikKel, Dirk (1988). «Data collection with Hand-held computers: Contributions to questionnaire design». Journal of Official Statistics, 4, 141-154.

Waterton, J.J. y Duffy, J.C. (1984). «A comparison of Computer Assisted Personal Interview and traditional methods in the collection of self-report alcohol consumption data in a field survey». International Statistical Review, 2, 173-182. <https://doi.org/10.2307/1403100>

Watson, Nicole y WiLKINS, Roger (2015). «Design Matters The Impact of CAPI on Interview Length». Field Methods, 27 (3), 244-264. <https://doi.org/10.1177/1525822X15584538>

West, Brady T. y Groves, Robert M. (2013). «A Propensity-Adjusted Interviewer Performance Indicator». Public Opinion Quarterly, 77 (3), 740-768. $<$ https://doi.org/10.1093/poq/nft002>

Zhang, Chan y ConraD, Frederic (2014). «Speeding in Web Surveys: The Tendency to answer fast and its association with straighlining». Survey Research Methods, 8 (2), 127-135.

Anexo 1. Temática de las preguntas del cuestionario (estudios 2893 y 2894)

\section{Rasgos sociodemográficos}

- Sexo.

- Edad alcanzada.

- Situación de convivencia actual del entrevistado.

- Estado civil.

- Máximo nivel de estudios alcanzado.

- Religiosidad y frecuencia de asistencia a oficios religiosos.

- Persona que aporta más ingresos al hogar.

— Situación laboral (relación con la actividad): trabajada, parados, jubilado, estudiante, trabajo doméstico no remunerado.

- Ocupación u oficio.

- Situación profesional: asalariado, empresario, autónomo, ayuda familiar, cooperativista, otros.

- Titularidad (privado/estatal) del lugar de trabajo (administración pública, empresa pública, empresa privada, organización sin ánimo de lucro, servicio doméstico y otros).

- Actividad principal de la empresa u organización donde trabaja la persona que aporta más ingresos al hogar.

- Clase social.

- Nacionalidad española (de nacimiento o adquirida con posterioridad).

- País de nacimiento.

— Número de teléfono (para comprobación de la entrevista). 


\section{Resto de preguntas del cuestionario}

- Evaluación de la situación económica y política de la Comunidad de Madrid y de España actualmente.

- Valoración retrospectiva de la labor que está llevando a cabo el actual gobierno autonómico de la Comunidad de Madrid (4 años): educación, sanidad, seguridad ciudadana...

- Valoración retrospectiva de la gestión del gobierno autonómico de la Comunidad de Madrid, de la política del gobierno central respecto a la Comunidad de Madrid (4 años) y de una posible gestión si gobernase el PSOE.

- Valoración de la gestión del gobierno central.

- Interés por la campaña electoral de las elecciones autonómicas de la Comunidad de Madrid de 2011.

- Utilidad de diversos aspectos de la campaña electoral: informarse sobre los programas, conocer a los candidatos y candidatas, ver las diferencias entre los partidos y decidir el voto.

- Frecuencia en el seguimiento de información política y electoral a través de medios de comunicación.

- P. 13: Frecuencia en el seguimiento de la campaña electoral en Internet.

- P. 13a: Tipología de páginas de Internet que consulta en el seguimiento de la campaña electoral.

- Frecuencia con la que ha hablado de las elecciones durante la campaña electoral con la familia y los amigos o amigas.

- Valoración de la campaña electoral de partidos políticos en las elecciones autonómicas de la Comunidad de Madrid de 2011.

- Forma de seguimiento de la campaña electoral: vio propaganda electoral por televisión, leyó folletos, cartas, etc.

- P. 17: Decisión de voto en las elecciones autonómicas de la Comunidad de Madrid de 2011.

- P. 17a: Partidos entre los que dudó en las elecciones autonómicas de la Comunidad de Madrid de 2011.

- P. 18 y 18a: Influencia y sentido de las movilizaciones del $15 \mathrm{M}$ en las elecciones autonómicas de la Comunidad de Madrid de 2011.

- P. 19: Participación electoral en las elecciones autonómicas de la Comunidad de Madrid de 2011.

- P. 19a, 19b y 19c: Razones para no ir a votar o que le impidieron ir en las elecciones autonómicas. Momento de la decisión de no votar.

- P. 20, 20a y 20b: Momento de la decisión de voto en las elecciones autonómicas de la Comunidad de Madrid de 2011. Recuerdo de voto en las elecciones autonómicas y razones para votar a algún partido político.

- Temas, autonómicos o nacionales, que más influyeron sobre su decisión de voto en las elecciones autonómicas de la Comunidad de Madrid de 2011.

- Decisión respecto de haber conocido antes los resultados de las elecciones autonómicas.

- Valoración de los resultados de las elecciones autonómicas de la Comunidad de Madrid de 2011. 
- Problemas que deberían ser más importantes para el gobierno autonómico de la Comunidad de Madrid.

- Preg. 25 y 25a: Participación electoral en las elecciones generales de marzo de 2008. Recuerdo de voto.

- Conocimiento y escala de valoración de líderes políticos nacionales y autonómicos: Esperanza Aguirre, Tomás Gómez, Gregorio Gordo, Luis de Velasco, Mariano Rajoy y José Luis Rodríguez Zapatero.

- Cercanía hacia partidos políticos de la Comunidad de Madrid: PP, PSOE, IU y UPyD.

- Acuerdo con distintas frases sobre la política: esté quien esté en el poder siempre busca sus intereses, los políticos y las políticas se preocupan de la gente como Ud., y la persona entrevistada está mejor informada que la mayoría de la gente.

- Sentimiento nacionalista de la Comunidad de Madrid.

- Preferencia entre diferentes alternativas de organización territorial del Estado en España.

- Preg. 32: Participación electoral en las elecciones municipales de la Comunidad de Madrid de 2011.

- Preg. 32a, 32b y 32c: Momento de la decisión de voto en las elecciones municipales de la Comunidad de Madrid de 2011. Recuerdo de voto en las elecciones municipales y razones para votar a algún partido político.

- Valoración de los resultados de las elecciones municipales de la Comunidad de Madrid de 2011.

- Autodefinición de su ideología política.

- Escala de autoubicación ideológica y de partidos políticos de la Comunidad de Madrid.

- Preg. 36a y 36b: Participación electoral y recuerdo de voto en las elecciones generales de 2008 y en las elecciones autonómicas de la Comunidad de Madrid de 2007.

Anexo 2. Distribución muestral considerando sexo, edad, tipo de familia, nivel de estudios, relación con la actividad, situación profesional y clase social (autoadscripción)

\begin{tabular}{llrrr}
\hline & & \multicolumn{3}{c}{ Modalidad } \\
\cline { 3 - 5 } & & Papel & Electrónica & Subtotal \\
\hline Sexo & Hombre & $47,6 \%$ & $47,9 \%$ & $47,7 \%$ \\
Chi cuadrado: 0,011 & Mujer & $52,4 \%$ & $52,1 \%$ & $52,3 \%$ \\
Signif.: 0,917 & Número de casos & 922 & 455 & 1.377 \\
\hline Edad por cuotas & $18-24$ años & $8,6 \%$ & $8,8 \%$ & $8,6 \%$ \\
Chi cuadrado: 0,360 & $25-34$ años & $22,0 \%$ & $22,0 \%$ & $22,0 \%$ \\
Signif.: 0,996 & $35-44$ años & $21,3 \%$ & $22,0 \%$ & $21,5 \%$ \\
& $45-54$ años & $17,9 \%$ & $16,7 \%$ & $17,5 \%$ \\
& $55-64$ años & $12,6 \%$ & $12,5 \%$ & $12,6 \%$ \\
& 65 y más años & $17,7 \%$ & $18,0 \%$ & $17,8 \%$ \\
& Número de casos & 922 & 455 & 1.377 \\
\hline
\end{tabular}


Anexo 2. Distribución muestral considerando sexo, edad, tipo de familia, nivel de estudios, relación con la actividad, situación profesional y clase social (autoadscripción) (continuación)

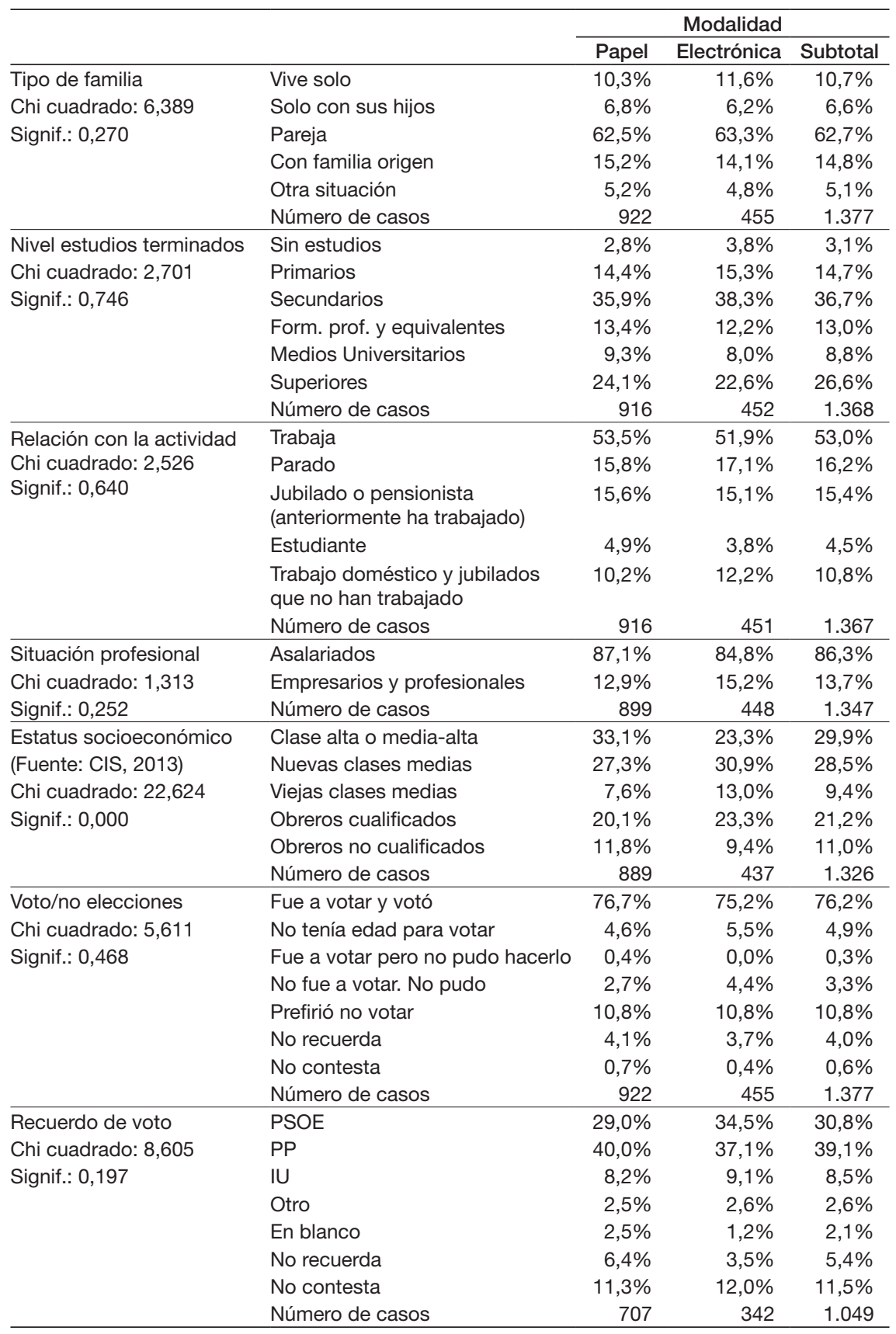

